\title{
Recoverability of Renal Function after Treatment of Adult Patients with Unilateral Obstructive Uropathy: A Prospective Study
}

\author{
Atef Badawy, Osama A. Abdel Gawad, Mohammed A. El-Shazly, Mahmoud H. Hewaidy* \\ Urology Department, Faculty of Medicine, Menoufia University, Menoufia, Egypt. \\ *Corresponding Author: Mahmoud H. Hewaidy, Mobile: +20 01009150509, E-mail: drmhh8383@ yahoo.com
}

\begin{abstract}
Background: Obstructive uropathy refers to any blockage of urine drainage at any level of the urinary tract. It may be chronic or acute and may occur unilateral or bilateral. The prediction of the recoverability of kidney function after treatment of unilateral obstructive uropathy is of great clinical value to the urologists and nephrologists as it may affect the decision of treatment.

Objectives: To detect changes in split renal function of unilaterally obstructed kidney after a period of 6 weeks of drainage.

Patients and methods: This prospective observational study included 66 adult patients divided into 2 groups; Group A included patients with split function less than $10 \%$ (36 patients) and Group B included patients with split function of $10 \%$ or more (30 patients).

Results: The study showed that there were an insignificant difference between the two groups in age, sex, cause of obstruction, laterality of obstruction, method of drainage and patient's presentation. Also, there was an insignificant difference between pre- and post-drainage for group A (P-value 0.142). While there was a highly significance difference between pre- and post-drainage for group B (P-value $<0.001$ ).

Conclusion: the preoperative split function of the obstructed kidney is the independent factor affecting the recoverability of renal function. Where kidneys with split function less than $10 \%$ are irreversibly damaged and the decision of treatment can be taken with no need to do drainage followed by repeating renal scan. While stabilization or improvement of renal function is expected after relief of obstruction in kidneys with split function of $10 \%$ or greater.
\end{abstract}

Keywords: Adult, Isotope renography, Obstructive uropathy, Recoverability, Split function, Unilateral.

\section{INTRODUCTION}

Obstructive uropathy refers to any blockage of urine drainage at any level of the urinary tract. Obstructive uropathy may be long-term disease (chronic) or occur suddenly (acute). As well, it can occur unilateral or bilateral ${ }^{(\mathbf{1})}$.

Unilateral obstruction may be caused by many urologic disorders either intrinsic or extrinsic. Intrinsic causes may be intraluminal such as stones, blood clots, papillary necrosis and fungus balls or intramural causes e.g., ureteropelvic junction obstruction (UPJO), tumors and ureteric stricture. Extrinsic causes such as aberrant vessel in UPJO, accidental ligation of ureter, pregnant uterus, tumours, retroperitoneal fibrosis or hematoma ${ }^{(2)}$.

The prediction of the recoverability of kidney function after treatment of unilateral obstructive uropathy is of great clinical value to the urologists and nephrologists as it may affect the decision of treatment (3).

There is an agreement that in obstructed kidney, if the radioisotope estimated split function of less than $10 \%$, this warrants nephrectomy. In practice, this critical decision is verified by some urologists through isotope scanning 6 weeks after drainage of the obstructed kidney for possible recoverability and assessment of the renal split function ${ }^{(4,5)}$.

Does it really matter to do isotope scanning and take the decision to repair or to remove only 6 weeks
Qa1 after drainage? In other words, does it have an impact on the decision? We aimed in this study to answer that question.

The aim of this study is to detect changes in split renal function of unilaterally obstructed kidney with normal contralateral kidney after a period of 6 weeks of drainage.

\section{PATIENTS AND METHODS}

This prospective study was carried out in the Urology Department of Menoufia University Hospitals. It included 66 patients divided into 2 groups; Group A included patients with split function less than $10 \%$ (36 patients) and Group B included patients with split function of $10 \%$ or more (30 patients). All cases were evaluated in the period from April 2018 to April 2020.

Inclusion criteria included adult patients (more than 16 years old) with unilateral obstructive uropathy (radiologically grade 3 and 4 hydronephrosis) and normal contralateral kidney (normal morphology and no backpressure or nephropathy in ultrasound and GFR is greater than $40 \mathrm{~mL} / \mathrm{min} / 1.73 \mathrm{~m}^{2}$ in renal scan).

Exclusion criteria included patients with bilateral obstruction, infants and children with ureteropelvic junction obstruction (UPJO), cases with radiological grade 1 and 2 hydronephrosis and cases 
with pyelonephritis as it may be the cause of impaired function.

All patients included in this study were subjected to the following; detailed medical history, clinical examination, laboratory evaluation including urine analysis and serum creatinine.

Radiological investigations included abdominopelvic ultrasonography (US) to detect the side and degree of hydronephrosis and evaluate the other kidney, and non-contrast computed tomography (NCCT) abdomen and pelvis to give detailed anatomic study and evaluate hydronephrosis, hydroureter and urolithiasis.

All the patients in this study underwent isotope renography with estimation of split function on presentation prior to drainage, the used tracer was Technetium-99m- diethylene triamine penta acetic acid (DTPA).

All cases underwent drainage of their obstructed kidneys either through PCN (guided by US) or ureteric stents.

Isotope renography was repeated 6 weeks after the drainage of the obstructed kidney to assess effect of unilateral obstruction on renal function by comparing split function prior drainage and 6 weeks after drainage.

\section{Ethical approval:}

An approval of the study was obtained from Menoufia University academic and ethical committee. Every patient signed an informed written consent for acceptance of the operation.

\section{Statistics analysis}

Data were collected, revised, coded and entered to the Statistical Package for the Social Sciences (IBM SPSS) version 23. The quantitative data were presented as mean, standard deviations (SD) and ranges when parametric and median and interquartile range (IQR) when data were found non-parametric. The comparison between groups with qualitative data were done by using Chi-square test. The comparison between two groups with quantitative data and parametric distribution were done by using independent t-test. The comparison between two paired groups with quantitative data were done by Wilcoxon Rank test. P $<0.05$ was considered significant.

\section{RESULTS}

The study included 50 men (75.8\%) and 16 women (24.2\%), with a mean age of $43.83 \pm 10.49$ years and ranged 23 to 67 years old. There was no statistically significant difference between the two groups regarding age and sex with (Figure 1).
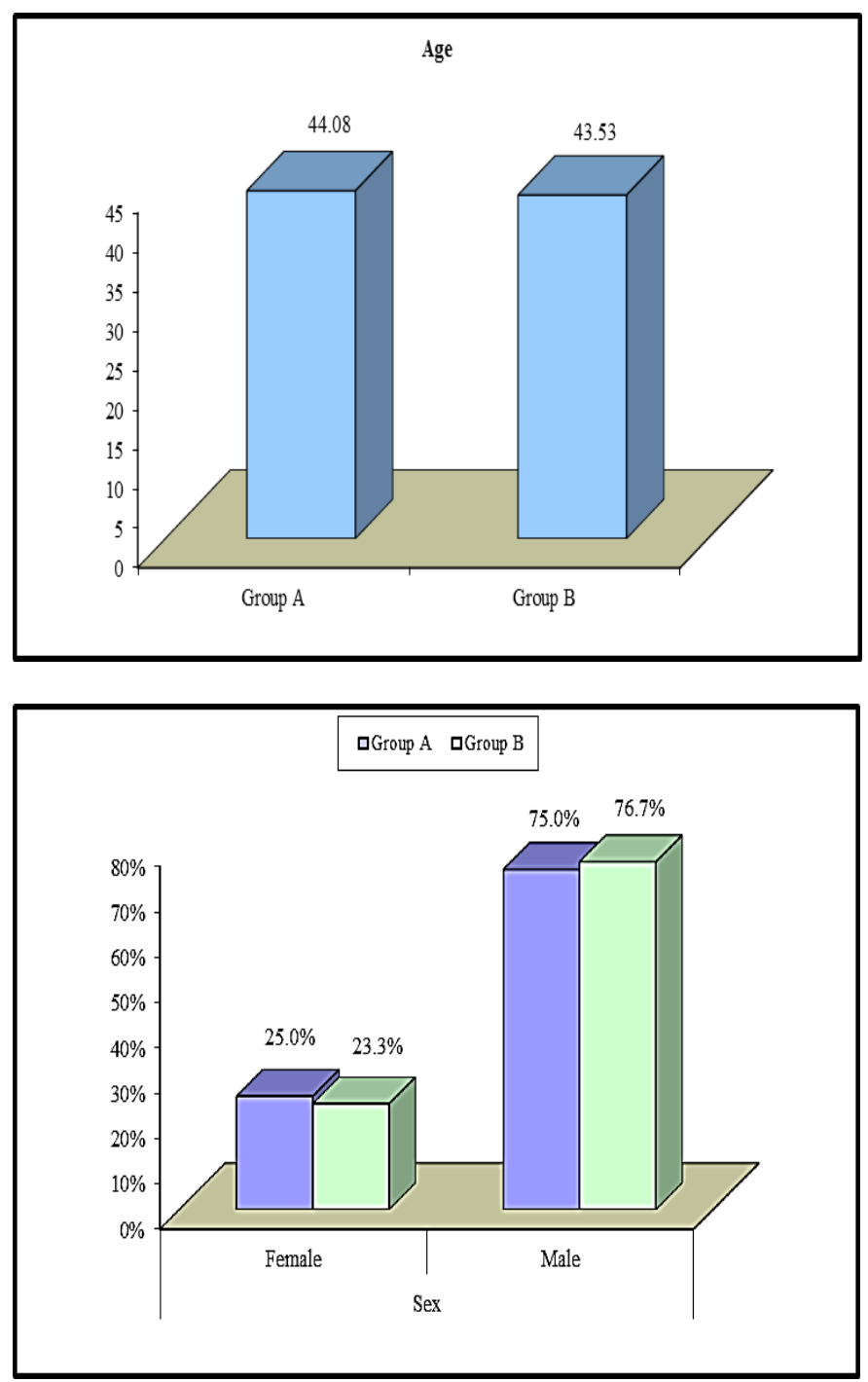

Fig. 1A

Fig. 1B

Figure (1a and b): Age and sex distribution in the two groups with no significant difference.

There was an insignificant difference between the two groups as regard the cause of obstruction (Table 1).

Table (1): Causes of obstruction in both groups.

\begin{tabular}{|l|c|c|c|c|c|c|l|}
\hline \multirow{2}{*}{ Cause of Obstruction } & \multicolumn{2}{|c|}{ Group A } & \multicolumn{2}{c|}{ Group B } & Test & P & \multirow{2}{*}{ Sig. } \\
\cline { 2 - 5 } & No. & value & No. & value & \\
\hline Ureteric stone & 20 & $55.6 \%$ & 13 & $43.3 \%$ & & & \\
Renal pelvic stone & 10 & $27.8 \%$ & 6 & $20.0 \%$ & 3.502 & 0.321 & NS \\
UPJ obstruction & 4 & $11.1 \%$ & 8 & $26.7 \%$ & & & \\
Stricture ureter & 2 & $5.5 \%$ & 3 & $10.0 \%$ & & & \\
\hline
\end{tabular}

$\mathrm{NS}=$ Non significant 
The most common presentation was loin pain. There was an insignificant difference between the two groups regarding patient's presentation, $\mathrm{p}$-value $=0.618$ (Figure 2).

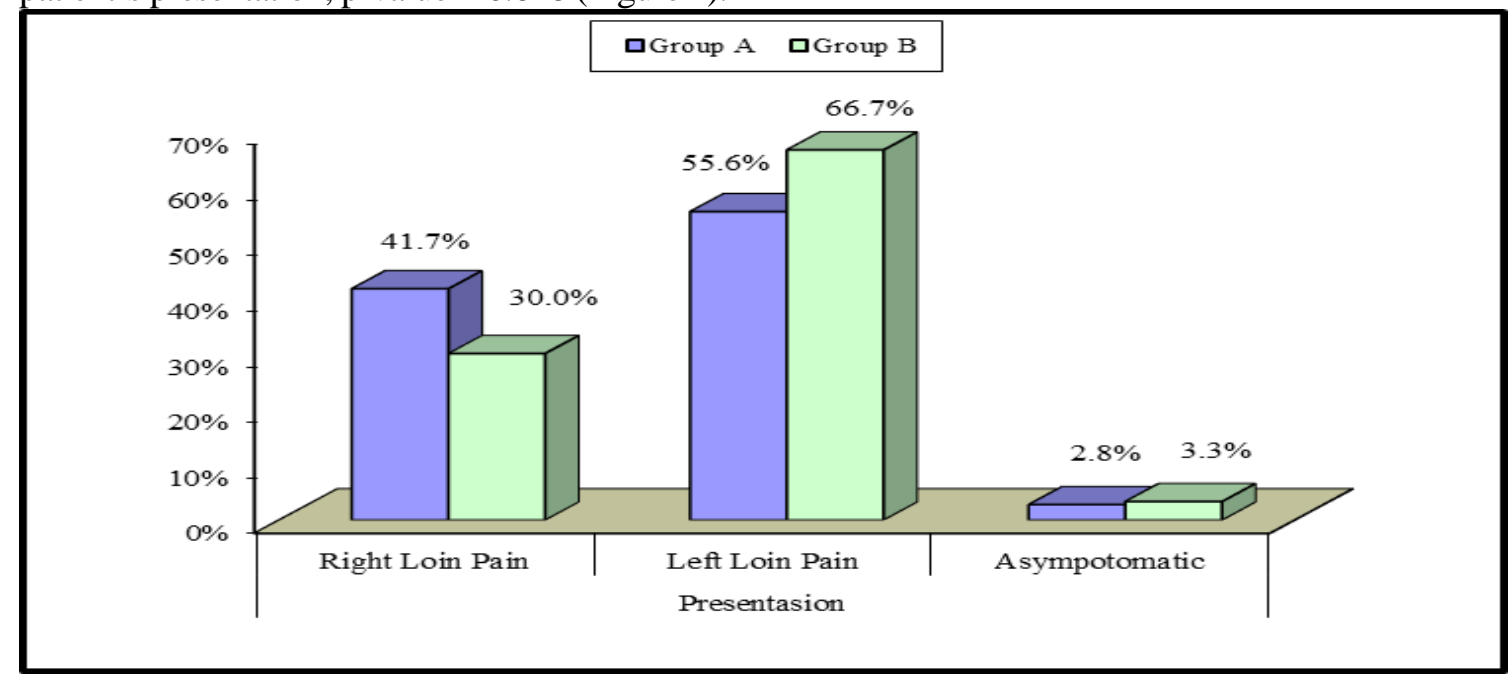

Figure (2): symptomatic and asymptomatic distribution.

All patients underwent drainage of their obstructed kidneys either through $\mathrm{PCN}$ or ureteric stents. There was an insignificant difference between the two groups regarding method of drainage, $p$-value $=0.323$ (Figure 3).

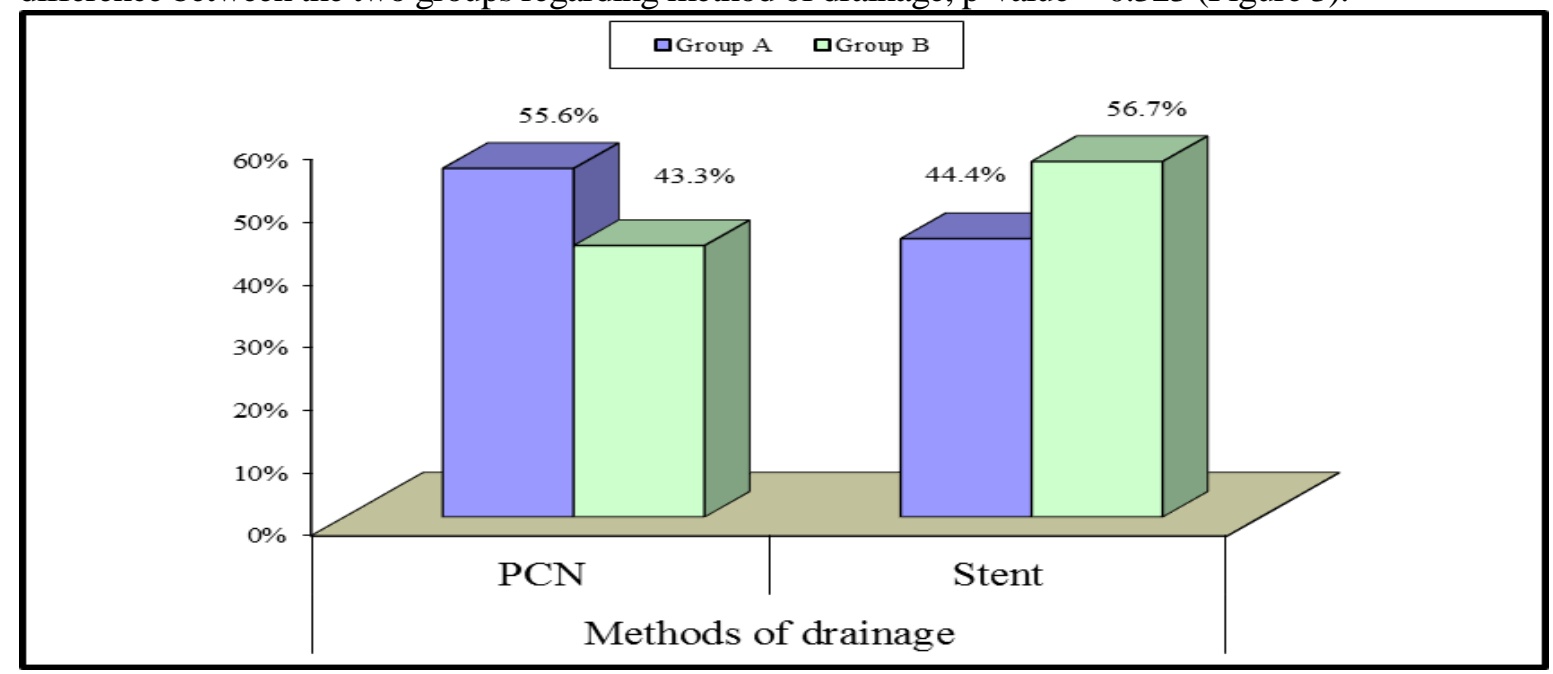

Figure (3): Methods of drainage in both groups.

There was a highly significant difference between pre- and post-drainage values of renographic split function (Table 2).

Table (2): Pre and post drainage split function

\begin{tabular}{|l|c|c|c|c|l|}
\hline \multirow{2}{*}{ Renogram } & Pre drainage & Post drainage & Test \\
\cline { 2 - 3 } & No. $\mathbf{6 6}$ & No. $\mathbf{6 6}$ & $\begin{array}{c}\text { P } \\
\text { value }\end{array}$ & Sig. \\
\hline Median (IQR) & $9(7-16)$ & $9 \begin{array}{c}(7-18) \\
4-45\end{array}$ & -4.154 & $<0.001$ & HS \\
Range (split function) & $3-34$ & & & &
\end{tabular}

$\mathrm{HS}=$ Highly significant $\mathrm{IQR}=$ Interquartile range

The comparison between preoperative and postoperative values of renographic split function showed that in group B there was a highly significant difference (Table 3 ).

Table (3): Pre and post drainage split function in each group.

\begin{tabular}{|l|l|c|c|c|c|c|l|}
\hline \multicolumn{2}{|c|}{ Renogram } & Pre drainage & Post drainage & $\begin{array}{c}\text { Mean } \\
\text { difference }\end{array}$ & Test value & P-value & Sig. \\
\hline Group A & $\begin{array}{l}\text { Median (IQR) } \\
\text { Range (split function) }\end{array}$ & $\begin{array}{c}7(6-8.5) \\
3-9\end{array}$ & $\begin{array}{c}7(6-8.5) \\
4-10\end{array}$ & $-0.22 \pm 0.87$ & -1.469 & 0.142 & NS \\
\hline Group B & $\begin{array}{l}\text { Median (IQR) } \\
\text { Range (split function) }\end{array}$ & $\begin{array}{c}16.5(14-20) \\
10-34\end{array}$ & $\begin{array}{c}21.5(14-32) \\
10-45\end{array}$ & $-4.80 \pm 4.98$ & -3.896 & $<0.001$ & HS \\
\hline
\end{tabular}




\section{DISCUSSION}

Unilateral urinary tract obstruction is a common disease, and can affect ipsilateral renal parenchyma up to total loss, painful or complicated non-functioning kidneys are best treated by simple nephrectomy ${ }^{(6)}$.

Relive of the early obstructed kidneys is beneficial, and results in improvement of renal function, however, determination of residual renal function of this obstructed kidney is difficult, and till now, no cut off point determine that ${ }^{(7)}$.

In our study we compared two groups, all of them had unilateral obstructed urinary tract; group A (36 patients) had split function below 10\%, and group B (30 patients) had split function of $10 \%$ or more. It was a prospective study, it was performed from April 2018 to April 2020. All patients were successfully drained either through PCN or ureteric stents. Then isotope renography was repeated 6 week after drainage of every patient.

While the prospective study of Khalaf $\boldsymbol{e t}$ al. ${ }^{(7)}$, included 91 consecutive adult patients, with unilateral renal obstruction and a normal contralateral kidney, between March 2000 to April 2002. All patients were treated using a variety of endoscopic and open surgical procedures. Then all patients were seen regularly at 3 , 6 , and 12 months for a mean follow-up of $13 \pm 6$ months. At each visit, US and renography were carried out, and excretory urography was performed at least once during follow-up.

Our study included 50 men and 16 women, with a mean age of $43.83 \pm 10.49$ (range 23 to 67 years). The causes of hydronephrosis were ureteral stones in 33 patients, renal pelvic stones in 16 patients, UPJ obstruction in 12 patients and stricture ureter in 5 patients. Of the patients, 64 had loin pain and two were pain free. The obstructed kidney was the right one in 26 patients and the left kidney in 40 patients. A total of 66 patients of the study, 33 patients (20 in group A and 13 in group $\mathrm{B}$ ) were drained through $\mathrm{PCN}$ and 33 patients (16 in group A and 17 in group B) were drained through ureteric stent.

A study of Khalaf et al. ${ }^{(7)}$, included 60 men and 31 women, with a mean age of $48 \pm 13$ years (range 21 to 65 years). The causes of hydronephrosis were ureteral stricture in 49 , ureteral stones in 24 , renal stones in 12, and ureteropelvic junction (UPJ) obstruction in 6 patients. Obstruction was relieved in all patients using a variety of endoscopic and open surgical procedures.

In our study, we estimated preoperative median values of renographic split function were 7 and postoperative median values 7 in group A with p-value of 0.142 . While in group $\mathrm{B}$, preoperative median values were 16.5 and postoperative median values were 21.5 with p-value of $<0.001$ (highly significant).
In the study of Khalaf et al. ${ }^{(7)}$, the mean preoperative and postoperative values of the selective renographic GFR were $20 \pm 12$ and $27 \pm 20$ respectively. They estimated a preoperative clearance value of 10 $\mathrm{mL} / \mathrm{min} / 1.73 \mathrm{~m}^{2}$ as the cutoff point to determine the best prediction of stabilization or improvement of renal function after the relief of obstruction, giving a sensitivity of $90 \%$ and specificity of $66 \%$. Of the 91 patients in the study, the baseline GFR of the ipsilateral kidney was greater than $10 \mathrm{~mL} / \mathrm{min} / 1.73 \mathrm{~m}^{2}$ in 66 and $10 \mathrm{~mL} / \mathrm{min} / 1.73 \mathrm{~m}^{2}$ or less in the remaining 25 patients.

In our study, only the preoperative selective renographic split function of the obstructed kidney sustained statistical significance as independent factor affecting renal functional recovery. While there were no statistically significant difference between the two groups regarding many factors e.g. age of the patient, patient's sex, patient's presentation, laterality of obstruction (right or left), cause of obstruction or method of drainage (PCN or ureteric stent).

In the study of Khalaf et al. ${ }^{(7)}$, on their multivariate analysis, only the preoperative selective renographic GFR and renal perfusion of the ipsilateral kidney sustained their statistical significance as independent factors affecting renal functional recovery.

Many factors can affect renal recovery after relief of obstruction such as the degree and duration of obstruction, the function of the contralateral kidney, patient age, pyelolymphatic backflow, compliance of the ureter and renal pelvis, presence of infection, and concurrent nephrotoxic agents or medications e.g. contrast material and non-steroidal analgesics ${ }^{(3)}$. But some of these factors cannot be studied except in an experimental situation, e.g. the degree and duration of obstruction, pyelolymphatic backflow, and compliance of the ureter and renal pelvis. In clinical practice, the presence of many interacting variables makes the prediction of the recovery of renal function difficult ${ }^{(7)}$.

Our study was designed to include only cases of unilateral obstruction with marked hydronephrosis (radiological grade 3 or 4 only) with a normal contralateral kidney to decrease the number of interacting variables, this allows more reliable interpretation of data. Additional studies included a larger number of patients are warranted to investigate the renal function recoverability in bilateral obstruction.

Previous reports used univariate analyses in their study of the methods of predicting the renal function recovery after the relief of obstruction ${ }^{(8-15)}$. In such analyses, each factor is studied separately, irrespective of the other relevant factors. Although this method is simple and transparent, but it doesn't take into account the interaction among all other corresponding variables. Although the multivariate analysis is accurate, it needs a large number of patients 
for proper use, particularly when the number of studied variables is numerous.

Our study have demonstrated that the preoperative renographic split function of the obstructed kidney is the only independent variable to predict for the recoverability of renal function after relief of advanced unilateral hydronephrosis. Other factors which are expected to have a significant influence on the recovery of renal function, e.g. parenchymal thickness, parenchymal echogenicity, and corticomedullary differentiation, should be statistically analyzed in additional studies with a larger number of patients.

We have confirmed the previous information that no recovery of renal function can be expected after the relief of obstruction if the relative renographic split function of the corresponding kidney has fallen to less than $10 \%$, but when the preoperative split function is $10 \%$ or greater, kidney function can improve considerably ${ }^{(3)}$.

However, these results may not be applicable to young children in whom the potential for recovery of what seems to be a severely damaged kidney is great. The recoverability of renal function in children needs additional prospective studies with a large number of cases. An almost general agreement has been reached among urologists that the better the renal blood flow, the better is the recuperation after relief of the obstruction. However, the critical value of renal blood flow which can predict for recovery of renal function has not yet been established ${ }^{(7)}$.

The limitation of our study is; the relatively small number of patients included in the study (66 patients), absence of data about duration of obstruction and absence of some ultrasonographic data of the obstructed kidney e.g. parenchymal thickness, echogenicity and corticomedullary differentiation, or data about other kidney which may show compensatory hypertrophy.

\section{CONCLUSION}

The preoperative split function of the obstructed kidney is the independent factor affecting recoverability of renal function in adult patients with unilateral obstructive uropathy, radiological grade 3 or 4 hydronephrosis and normal contralateral kidney.

Where kidneys with split function less than $10 \%$ are irreversibly damaged and the decision of treatment can be taken with no need to do drainage followed by repeating renal scan. While stabilization or improvement of renal function is expected after relief of obstruction in kidneys with split function of $10 \%$ or greater.

\section{REFERENCES}

1. Mujoomdar M, Dionne F, Moulton $\mathrm{K}$ et al. (2012): Optimizing Health System Use of Medical Isotopes and Other Imaging Modalities. APPENDIX 2.17, Suspected Obstructive Uropathy. Ottawa (ON): Canadian Agency for Drugs and Technologies in Health. https://www.ncbi.nlm.nih.gov/books/NBK174872/.

2. Zeidel M (2012): Obstructive Uropathy. Goldman's Cecil Medicine. Twenty-Fourth Edition: Pp. 776-780 https://www.sciencedirect.com/book/9781437716047/gol dmans-cecil-medicine

3. Shokeir A, Provoost A, Nijman R (1999): Recoverability of renal function after relief of chronic partial upper urinary tract obstruction. BJU international, 83(1):11-7.

4. Coronan J (1991): Contemporary concepts in imaging urinary tract obstruction. Radiol Clin North Am., 29:52742.

5. JY G (1992): The pathophysiology of urinary obstruction. In Walsh PC, Gittes, Perlumutter, Stamey, eds. Campbell's Urology 5th edn. Philadelphia: Saunders Co., Pp. 499-532.

6. Yang M, Gao F, Liu H et al. (2013): Prospectively estimating the recoverability of renal function after relief of unilateral urinary obstruction by measurement of renal parenchymal volume. Academic Radiology, 20(4):401-6.

7. Khalaf I, Shokeir A, El-Gyoushi F et al. (2004): Recoverability of renal function after treatment of adult patients with unilateral obstructive uropathy and normal contralateral kidney: a prospective study. Urology, 64(4):664-8.

8. Kalika V, Bard RH, Iloreta A et al. (1981): Prediction of renal functional recovery after relief of upper urinary tract obstruction. The Journal of Urology, 126(3):301-4.

9. Lome L, Pinsky S, Levy L (1979): Dynamic renal scan in the non-visualizing kidney. The Journal of Urology, 121(2):148-52.

10. Sherman R, Blaufox $M$ (1980): Obstructive uropathy in patients with nonvisualization on renal scan. Nephron, 25(2):82-6.

11. Chibber P, Chisholm G, Hargreave $T$ et al. (1981): 99mTechnetium DMSA and the prediction of recovery in obstructive uropathy. British Journal of Urology, 53(6):492-5.

12. Belis J, Belis T, Lai J et al. (1982): Radionuclide determination of individual kidney function in the treatment of chronic renal obstruction. The Journal of Urology, 127(4):636-41.

13. Huland $H$, Gonnermann $D$, Werner B et al. (1988): A new test to predict reversibility of hydronephrotic atrophy after stable partial unilateral ureteral obstruction. J Urol., 140: 1591-1594.

14. Ransley P, Dhillon H, Gordon I et al. (1990): The postnatal management of hydronephrosis diagnosed by prenatal ultrasound. The Journal of Urology, 144(2):5847.

15. HK D (1988): Prenatally diagnosed hydronephrosis: the Great Ormond Street experience. Br J Urol., 81:39-44. 